This is a self-archived - parallel published version of this article in the publication archive of the University of Vaasa. It might differ from the original.

\title{
Prediction law of fractional Brownian motion
}

Author(s): Sottinen, Tommi; Viitasaari, Lauri

Title: $\quad$ Prediction law of fractional Brownian motion

Year: $\quad 2017$

Version: Accepted manuscript

Copyright C)2017 Elsevier. Creative Commons AttributionNonCommercial-NoDerivatives 4.o International (CC BY-NCND 4.o) lisence, https://creativecommons.org/licenses/by-ncnd/4.0/deed.en

Please cite the original version:

Sottinen, T., \& Viitasaari, L., (2017). Prediction law of fractional Brownian motion. Statistics and probability letters 129, 155166. https://doi.org/10.1016/j.spl.2017.05.006 


\title{
PREDICTION LAW OF FRACTIONAL BROWNIAN MOTION
}

\author{
TOMMI SOTTINEN \\ Department of Mathematics and Statistics, University of Vaasa, P.O. Box 700, \\ FIN-65101 Vaasa, FINLAND
}

\section{LAURI VIITASAARI}

Department of Mathematics and System Analysis, Aalto University School of Science, Helsinki, P.O. Box 11100, FIN-00076 Aalto, FINLAND

\begin{abstract}
We calculate the regular conditional future law of the fractional Brownian motion with index $H \in(0,1)$ conditioned on its past. We show that the conditional law is continuous with respect to the conditioning path. We investigate the path properties of the conditional process and the asymptotic behavior of the conditional covariance.
\end{abstract}

\section{INTRODUCTION}

Let $B^{H}=\left(B_{t}^{H}\right)_{t \in \mathbb{R}_{+}}$be the fractional Brownian motion with Hurst index $H \in(0,1)$. Let $u \in \mathbb{R}_{+}$, and let $\mathscr{F}_{u}$ be the $\sigma$-field generated by the fractional Brownian motion on the interval $[0, u]$. We study the prediction of $\left(B_{t}\right)_{t \geq u}$ given the information $\mathscr{F}_{u}$. In other words, we study the conditional regular law of $\hat{B}^{H}(u)=B^{H} \mid \mathscr{F}_{u}$. It is well-known that such regular conditional laws for Gaussian processes exists and they are Gaussian with random conditional mean and deterministic conditional variance, see, e.g., Bogachev [4, Section 3.10] or Janson [6, Chapter 9]. Recently, LaGatta [8] introduced the notion of continuous disintegration. In our case it reads as follows: Let $T>0$ be arbitrary and let $\mathbb{P}_{T}$ be the law of the fractional Brownian motion on $[0, T]$. The regular conditional law $\mathbb{P}_{T}^{y}=\mathbb{P}_{T}\left[\cdot \mid B_{v}^{H}=y(v), v \leq u\right]$ is continuous with respect to $y$ if $y_{n} \rightarrow y$ (in sup-norm) implies $\mathbb{P}_{T}^{y_{n}} \rightarrow \mathbb{P}_{T}^{y}$ (weakly). We calculate the regular conditional law of fractional Brownian motion explicitly and show that it is continuous with respect to the conditioning trajectory.

Perhaps the earliest result on the prediction for fractional Brownian motion is due to Gripenberg and Norros [5]. They provided the conditional mean of the fractional

E-mail addresses: tommi.sottinen@iki.fi, lauri.viitasaari@aalto.fi.

Date: November 22, 2016.

2010 Mathematics Subject Classification. 60G22; 60G25.

Key words and phrases. Fractional Brownian motion; prediction; regular conditional law.

T. Sottinen was partially funded by the Finnish Cultural Foundation (National Foundations' Professor Pool).

L.Viitasaari was partially funded by the Emil Aaltonen Foundation. 
Brownian motion with parameter $H>\frac{1}{2}$ based on observations extending to the infinite past. Norros et al. [10] provided the conditional mean for the whole range $H \in(0,1)$ based on the observations on a compact interval. While the conditional expectation of the fractional Brownian motion is well understood, it seems that the regular conditional law has not been studied at all.

\section{Preliminaries}

We recall some facts for fractional Brownian motion and fractional calculus. As general references for fractional Brownian motion we refer to Biagini et al. [3] and Mishura [9]. The standard reference for fractional calculus is Samko et al. [12].

The fractional Brownian motion $B^{H}=\left(B_{t}^{H}\right)_{t \in \mathbb{R}_{+}}$with Hurst index $H \in(0,1)$ is the centered Gaussian process with covariance

$$
r_{H}(t, s)=\frac{1}{2}\left[t^{2 H}+s^{2 H}-|t-s|^{2 H}\right] .
$$

The case $H>\frac{1}{2}$ corresponds to the long-range dependent case, or positively correlated increments. The case $H<\frac{1}{2}$ corresponds to the short-range dependent case, or negatively correlated increments. For $H=\frac{1}{2}$, we have the classical Brownian motion.

Let

$$
I_{t-}^{\alpha}[f](s):=\frac{1}{\Gamma(\alpha)} \int_{s}^{t} f(z)(z-s)^{\alpha-1} \mathrm{~d} z, \quad s \in(0, t),
$$

be the right-sided fractional integral of order $\alpha \in(0,1)$. The inverse of $I_{t-}^{\alpha}$ is the right-sided fractional derivative

$$
I_{t-}^{-\alpha}[f](s):=-\frac{1}{\Gamma(1-\alpha)} \frac{\mathrm{d}}{\mathrm{d} s} \int_{s}^{t} f(z)(z-s)^{-\alpha} \mathrm{d} z .
$$

For $u \in \mathbb{R}_{+}$, define

$$
\mathfrak{K}_{H, u}[f](t):=\sigma_{H} t^{H-\frac{1}{2}} I_{u-}^{H-\frac{1}{2}}\left[(\cdot)^{H-\frac{1}{2}} f\right](t),
$$

where

$$
\sigma_{H}=\sqrt{\frac{\pi\left(H-\frac{1}{2}\right) 2 H}{\Gamma(2-2 H) \sin \left(\pi\left(H-\frac{1}{2}\right)\right)}} .
$$

Let $\mathfrak{K}_{H, u}^{-1}$ be the inverse of $\mathfrak{K}_{H, u}$, i.e.,

$$
\mathfrak{K}_{H, u}^{-1}[f](t)=\frac{1}{\sigma_{H}} t^{\frac{1}{2}-H} I_{u-}^{\frac{1}{2}-H}\left[(\cdot)^{H-\frac{1}{2}} f\right](t) .
$$

Indicator functions $\mathbf{1}_{[0, t)}$ belong to the domains of $\mathfrak{K}_{H, t}$ and $\mathfrak{K}_{H, t}^{-1}$ for all $t>0$ and $H \in(0,1)$. So, we can define

$$
\begin{aligned}
k_{H}(t, s) & :=\mathfrak{K}_{H, t}\left[\mathbf{1}_{[0, t)}\right](s), \\
k_{H}^{-1}(t, s) & :=\mathfrak{K}_{H, t}^{-1}\left[\mathbf{1}_{[0, t)}\right](s) .
\end{aligned}
$$

Then

$$
k_{H}(t, s)=d_{H}\left[\left(\frac{t}{s}\right)^{H-\frac{1}{2}}(t-s)^{H-\frac{1}{2}}-\left(H-\frac{1}{2}\right) s^{\frac{1}{2}-H} \int_{s}^{t} z^{H-\frac{3}{2}}(z-s)^{H-\frac{1}{2}} \mathrm{~d} z\right],
$$


where

$$
d_{H}=\sqrt{\frac{2 H \Gamma\left(\frac{3}{2}-H\right)}{\Gamma\left(H+\frac{1}{2}\right) \Gamma(2-2 H)}} .
$$

(A similar formula can be found for $k_{H}^{-1}$ also, but we have no need for it here.)

Lemma 2.1 (Volterra Correspondence). Let $B^{H}$ be a fractional Brownian motion. Then the process

$$
W_{t}=\int_{0}^{t} k_{H}^{-1}(t, s) \mathrm{d} B_{s}^{H}
$$

is a Brownian motion. Moreover, the fractional Brownian motion can be recovered from it by

$$
B_{t}^{H}=\int_{0}^{t} k_{H}(t, s) \mathrm{d} W_{s}
$$

The Volterra correspondence of Lemma 2.1 above extends to a transfer principle of Lemma 2.2 below. We note that in Lemma 2.1 can be taken as the definition of an abstract Wiener integral with respect to the fractional Brownian motion. We refer to Pipiras and Taqqu [11] for details on Wiener integration with respect to fractional Brownian motions, and for [13] for a more general discussion on abstract Wiener integration.

Lemma 2.2 (Transfer Principle). Let $B^{H}$ and $W$ be as in Lemma 2.1. Then, for all $u \in \mathbb{R}_{+}$,

$$
\int_{0}^{u} f(t) \mathrm{d} W_{t}=\int_{0}^{u} \mathfrak{K}_{H, u}^{-1}[f](t) \mathrm{d} B_{t}^{H}
$$

for all $f \in L^{2}([0, u])$, and

$$
\int_{0}^{u} f(t) \mathrm{d} B_{t}^{H}=\int_{0}^{u} \mathfrak{K}_{H, u}[f](t) \mathrm{d} W_{t},
$$

for all $f \in \mathfrak{K}_{H, u}^{-1} L^{2}([0, u])$.

\section{Regular Conditional Law}

Theorem 3.1 (Prediction Law). The conditional process $\hat{B}^{H}(u)=\left(\hat{B}_{t}^{H}(u)\right)_{t \geq u}$ is Gaussian with $\mathscr{F}_{u}$-measurable mean function

$$
\hat{m}_{t}^{H}(u)=B_{u}^{H}-\int_{0}^{u} \Psi_{H}(t, s \mid u) \mathrm{d} B_{s}^{H},
$$

where

$$
\Psi_{H}(t, s \mid u)=-\frac{\sin \left(\pi\left(H-\frac{1}{2}\right)\right)}{\pi} s^{\frac{1}{2}-H}(u-s)^{\frac{1}{2}-H} \int_{u}^{t} \frac{z^{H-\frac{1}{2}}(z-u)^{H-\frac{1}{2}}}{z-s} \mathrm{~d} z,
$$

and deterministic covariance function

$$
\hat{r}_{H}(t, s \mid u)=r_{H}(t, s)-\int_{0}^{u} k_{H}(t, v) k_{H}(s, v) \mathrm{d} v .
$$

Moreover, the regular conditional law is continuous with respect to the conditioning trajectory $\left(B_{v}^{H}\right)_{v \leq u}$. 
Proof. Let $B^{H}$ and $W$ be as in Lemma 2.1. Let $t \geq u$. Then

$$
\begin{aligned}
\hat{m}_{t}(u) & =\mathbb{E}\left[B_{t}^{H} \mid \mathscr{F}_{u}\right] \\
& =\mathbb{E}\left[\int_{0}^{t} k_{H}(t, s) \mathrm{d} W_{s} \mid \mathscr{F}_{u}^{W}\right] \\
& =\int_{0}^{u} k_{H}(t, s) \mathrm{d} W_{s} \\
& =\int_{0}^{u} k_{H}(u, s) \mathrm{d} W_{s}-\int_{0}^{u}\left[k_{H}(u, s)-k_{H}(t, s)\right] \mathrm{d} W_{s} \\
& =B_{u}^{H}-\int_{0}^{u}\left[k_{H}(u, s)-k_{H}(t, s)\right] \mathrm{d} W_{s} .
\end{aligned}
$$

It remains to show that the function $s \mapsto k_{H}(u, s)-k_{H}(t, s), s \in[0, u]$, belongs to $\mathfrak{K}_{H, u}^{-1} L^{2}([0, u])$ and then to apply Lemma 2.2 and calculate the transfered kernel for the equation

$$
\int_{0}^{u}\left[k_{H}(u, s)-k_{H}(t, s)\right] \mathrm{d} W_{s}=\int_{0}^{u} \mathfrak{K}_{H, u}^{-1}\left[k_{H}(u, \cdot)-k_{H}(t, \cdot)\right](s) \mathrm{d} B_{s}^{H} .
$$

This was done in Pipiras and Taqqu [11, Theorem 7.1].

Let us then calculate the conditional covariance. Let $W$ be as before. Then

$$
\begin{aligned}
\hat{r}_{H}(t, s \mid u)= & \mathbb{E}\left[\left(B_{t}^{H}-\hat{m}_{t}(u)\right)\left(B_{s}^{H}-\hat{m}_{s}(u)\right) \mid \mathscr{F}_{u}\right] \\
= & \mathbb{E}\left[\left(\int_{0}^{t} k_{H}(t, v) \mathrm{d} W_{v}-\int_{0}^{u} k_{H}(t, v) \mathrm{d} W_{v}\right) \times\right. \\
& \left.\left(\int_{0}^{s} k_{H}(s, w) \mathrm{d} W_{w}-\int_{0}^{u} k_{H}(s, w) \mathrm{d} W_{w}\right) \mid \mathscr{F}_{u}^{W}\right] \\
= & \mathbb{E}\left[\int_{u}^{t} k_{H}(t, v) \mathrm{d} W_{v} \int_{u}^{s} k_{H}(s, w) \mathrm{d} W_{w} \mid \mathscr{F}_{u}^{W}\right] \\
= & \int_{u}^{t \wedge s} k_{H}(t, v) k_{H}(s, v) \mathrm{d} v \\
= & r_{H}(t, s)-\int_{0}^{u} k_{H}(t, v) k_{H}(s, v) \mathrm{d} v,
\end{aligned}
$$

where the kernel $k_{H}(t, s)$ is given by $(2.1)$.

Finally, to invoke [8, Theorem 2.4], we must show that

$$
\sup _{v \in[0, u]} \frac{\sup _{t \in[0, T]}\left|r_{H}(v, t)\right|}{\sup _{w \in[0, u]}\left|r_{H}(v, w)\right|}<\infty
$$

for all $T>0$. Since $r_{H}$ is continuous,

$$
\frac{\sup _{t \in[0, T]}\left|r_{H}(v, t)\right|}{\sup _{w \in[0, u]}\left|r_{H}(v, w)\right|}=\frac{\left|r_{H}\left(v, t_{T, v}^{*}\right)\right|}{\left|r_{H}\left(v, w_{u, v}^{*}\right)\right|} \leq \frac{\left|r_{H}\left(v, t_{T, v}^{*}\right)\right|}{\left|r_{H}(v, v)\right|} .
$$

The ratio above is obviously bounded for all $v \in[\varepsilon, u]$ for any $\varepsilon>0$. As for $v \rightarrow 0$,

$$
\limsup _{v \rightarrow 0} \frac{\left|r_{H}\left(v, t_{T, v}^{*}\right)\right|}{\left|r_{H}(v, v)\right|} \leq 1
$$

since $r_{H}\left(v, t_{T, v}^{*}\right)=\sup _{t \in[0, T]} r_{H}(v, t) \geq r_{H}(v, v)$. 
Remark 3.1 (Brownian Motion). For $H=\frac{1}{2}$, we have $k_{\frac{1}{2}}(t, s)=\mathbf{1}_{[0, t)}(s)$ and $\mathfrak{K}_{\frac{1}{2}, u}$ is the identity operator. Consequently, we recover from the proof of Theorem 3.1 that

$$
\begin{aligned}
\hat{m}_{t}^{\frac{1}{2}}(u) & =W_{u}, \\
\hat{r}_{\frac{1}{2}}(t, s \mid u) & =t \wedge s-u .
\end{aligned}
$$

Remark 3.2 (Prediction Martingale). The formula (3.1) for the conditional expectation $\hat{m}_{t}^{H}(u)$ is rather complicated. Let us note, however, that for each fixed prediction horizon $t>0$, the process $\hat{m}_{t}^{H}(\cdot)$ is a Gaussian martingale on $[0, t]$ with bracket

$$
\mathrm{d}\left\langle\hat{m}_{t}(\cdot)\right\rangle_{u}=k_{H}(t, u)^{2} \mathrm{~d} u .
$$

Next we investigate the conditional covariance $\hat{r}_{H}(t, s \mid u)$ for fixed $s \leq t$ as a function of $u \in(0, s)$. The proofs are rather technical and lengthy. For this reason they are postponed into Section 4.

Proposition 3.1 (Conditional Covariance). $\hat{r}_{H}(t, s \mid \cdot)$ is infinitely differentiable and strictly decreasing on $(0, s)$ for any $H \in(0,1)$. For $H \in\left[\frac{1}{2}, 1\right)$ it is also convex.

Remark 3.3 (Short-Range Dependent Conditional Covariance). For $H \in\left(0, \frac{1}{2}\right)$, $\hat{r}_{H}(t, s \mid \cdot)$ is neither convex nor concave. Indeed, it can be shown that for $H \in\left(0, \frac{1}{2}\right)$, the kernel $k_{H}$ is positive and

$$
\lim _{s \rightarrow 0+} k_{H}(t, s)=\lim _{s \rightarrow t-} k_{H}(t, s)=\infty .
$$

Therefore

is neither increasing nor decreasing in $u$.

$$
\frac{\partial}{\partial u} \hat{r}_{H}(t, s \mid u)=-k_{H}(t, u) k(s, u)
$$

Proposition 3.2 (No-Information Asymptotics). Let $t \geq s$ be fixed.

(i) For $H<\frac{1}{2}$ we have, as $u \rightarrow 0$,

$$
\hat{r}_{H}(t, s \mid u)=r_{H}(t, s)-C_{H} u^{2 H}+o\left(u^{2 H}\right),
$$

where

$$
C_{H}=\frac{d_{H}^{2}}{2 H}\left(H-\frac{1}{2}\right)^{2}\left(\int_{1}^{\infty} w^{H-\frac{3}{2}}(w-1)^{H-\frac{1}{2}} \mathrm{~d} w\right)^{2} .
$$

(ii) For $H>\frac{1}{2}$ we have, as $u \rightarrow 0$,

$$
\hat{r}_{H}(t, s \mid u)=r_{H}(t, s)-C_{H, t, s} u^{2-2 H}+o\left(u^{2-2 H}\right),
$$

where

$$
C_{H, t, s}=\frac{d_{H}^{2}(t s)^{2 H-1}}{8-8 H} .
$$

Remark 3.4. It is interesting to note in Proposition 3.2 the different asymptotic behavior for the long-range dependent case $\left(H>\frac{1}{2}\right)$ and the short-range dependent case $\left(H<\frac{1}{2}\right)$. Indeed, for the long-range dependent case the principal term in the "remaining covariance" $r_{H}(t, s)-\hat{r}_{H}(t, s \mid u)$ is $C_{H} u^{2 H}$, where the constant $C_{H}$ is independent of $t$ and $s$. In the short-range dependent case the principal term is $C_{H, t, s} u^{2-2 H}$. So, the power reverts from $2 H$ to $2-2 H$ (and, consequently, the principal term remains convex) and the constant depends on $t$ and $s$. 
Proposition 3.3 (Full-Information Asymptotics). Let $H \in(0,1) \backslash\left\{\frac{1}{2}\right\}$. Then

(i) for $t=s$, we have, as $u \rightarrow s$,

$$
\hat{r}_{H}(s, s \mid u)=\frac{d_{H}^{2}}{2 H}(s-u)^{2 H}+o\left((s-u)^{2 H}\right),
$$

(ii) for $t>s$ we have, as $u \rightarrow s$,

$$
\hat{r}_{H}(t, s \mid u)=C_{H, t, s}(s-u)^{H+\frac{1}{2}}+o\left((s-u)^{H+\frac{1}{2}}\right),
$$

where

$$
C_{H, t, s}=\frac{d_{H}^{2}}{H+\frac{1}{2}}\left[\left(\frac{t}{s}\right)^{H-\frac{1}{2}}(t-s)^{H-\frac{1}{2}}+\left(\frac{1}{2}-H\right) s^{H-\frac{1}{2}} \int_{1}^{\frac{t}{s}} w^{H-\frac{3}{2}}(w-1)^{H-\frac{1}{2}}\right] .
$$

Finally, we examine the sample path continuity of the conditional process. Recall that a process $X=\left(X_{t}\right)_{t \in \mathbb{R}_{+}}$is Hölder continuous of order $\gamma$, if for all $T>0$ there exists an almost surely finite random variable $C_{T}$ such that

$$
\left|X_{t}-X_{s}\right| \leq C_{T}|t-s|^{\gamma}
$$

for all $t, s \leq T$. The Hölder index of the process is the supremum of all $\gamma$ such that (3.3) holds.

Next we show that the Hölder index of the conditional process $\hat{B}^{H}(u)$ and the conditional mean $\hat{m}^{H}(u)$ are the same as that of the fractional Brownian motion $B^{H}$. This is very important e.g. for pathwise stochastic analysis.

Proposition 3.4 (Hölder Continuity). Let $u>0$ be fixed. Then the conditional process $\hat{B}^{H}(u)$ and the conditional mean $\hat{m}^{H}(u)$ both have Hölder index $H$.

Proof. Let us first consider the conditional mean $\hat{m}^{H}(u)$. Since

$$
\hat{m}_{t}^{H}(u)=\int_{0}^{u} k_{H}(t, v) \mathrm{d} W_{v},
$$

we have, by the Itô isometry,

$$
\mathbb{E}\left[\left(\hat{m}_{t}^{H}(u)-\hat{m}_{s}^{H}(u)\right)^{2}\right]=\int_{0}^{u}\left[k_{H}(t, v)-k_{H}(s, v)\right]^{2} \mathrm{~d} v .
$$

Let $s \leq t$. By Lemma 2.1 and the Itô isometry, we have

$$
|t-s|^{2 H}=\int_{0}^{t}\left[k_{H}(t, v)-k_{H}(s, v)\right]^{2} \mathrm{~d} v .
$$

Thus

$$
\mathbb{E}\left[\left(\hat{m}_{t}^{H}(u)-\hat{m}_{s}^{H}(u)\right)^{2}\right] \leq|t-s|^{2 H}
$$

from which it follows, by the Kolmogorov continuity criterion, that $\hat{m}_{t}^{H}(u)$ is Hölder continuous of any order $\gamma<H$. Next we show that $\hat{m}_{t}^{H}(u)$ cannot be Hölder continuous of any order $\gamma>H$ at $t=u$. Since

$$
\hat{r}_{H}(t, t \mid u)=\int_{u}^{t}\left[k_{H}(t, v)\right]^{2} \mathrm{~d} v,
$$

Proposition 3.3 gives

$$
\int_{u}^{t}\left[k_{H}(t, v)\right]^{2} \mathrm{~d} v=\frac{d_{H}^{2}}{2 H}|t-u|^{2 H}+o\left(|t-u|^{2 H}\right) .
$$


Now it can be shown that for $H \neq \frac{1}{2}$ we have $\frac{d_{H}^{2}}{2 H}<1$, and hence we also have

$$
\int_{0}^{u}\left[k_{H}(t, v)-k_{H}(u, v)\right]^{2} \mathrm{~d} v=\left(1-\frac{d_{H}^{2}}{2 H}\right)(t-u)^{2 H}+o\left(|t-u|^{2 H}\right) .
$$

In particular, this shows that

$$
\mathbb{E}\left[\left(\hat{m}_{t}^{H}(u)-\hat{m}_{u}^{H}(u)\right)^{2}\right]=\int_{0}^{u}\left[k_{H}(t, v)-k_{H}(u, v)\right]^{2} \mathrm{~d} v \geq c_{H}|t-u|^{2 H} .
$$

Consequently, the claim follows from the sharpness of the Kolmogorov continuity criterion for Gaussian processes (see [1]).

Let us then consider the conditional process $\hat{B}^{H}(u)$. Since the conditional mean $\hat{m}^{H}(u)$ is Hölder continuous with index $\gamma$ if and only if $\gamma<H$, we may consider the centered conditional process $\bar{B}^{H}(u)=\hat{B}^{H}(u)-\hat{m}^{H}(u)$. Since

$$
\mathbb{E}\left[\left(\bar{B}_{t}^{H}(u)-\bar{B}_{s}^{H}(u)\right)^{2}\right]=|t-s|^{2 H}-\int_{0}^{u}\left[k_{H}(t, v)-k_{H}(s, v)\right]^{2} \mathrm{~d} v,
$$

the claim follows with the same arguments as in the conditional mean case.

\section{Proofs of Propositions 3.1, 3.2 and 3.3}

Proof of Proposition 3.1. Let $u<s \leq t$. From (3.2) we observe that

$$
\frac{\partial}{\partial u} \hat{r}_{H}(t, s \mid u)=-k_{H}(t, u) k_{H}(s, u) .
$$

Since $k_{H}(t, u)$ is infinitely differentiable with respect to $u$ for all $t$, it follows that $\hat{r}_{H}(t, s \mid u)$ is infinitely differentiable in $u$. Furthermore, since $k_{H}(t, u)>0$ for all $t$ and $u$, we observe that $\frac{\partial}{\partial u} \hat{r}_{H}(t, s \mid u)<0$. Hence $\hat{r}_{H}(t, s \mid u)$ is strictly decreasing in $u$.

Next we prove the convexity in $u$ of $\hat{r}_{H}(t, s \mid u)$ for $H>\frac{1}{2}$. For this it is sufficient to show that $-k_{H}(t, u) k_{H}(s, u)$ is increasing in $u$. Hence, it suffices to show that $k_{H}(t, s)$ is decreasing in $s$. Indeed, then $-k_{H}(t, u) k_{H}(s, u)$ is increasing in $u$ since $k_{H}(t, s) \geq 0$. By [7, Eq. (1.2)] we have

$$
k_{H}(t, s)=C_{H}(t-s)^{H-\frac{1}{2}} F\left(\frac{1}{2}-H, H-\frac{1}{2}, H+\frac{1}{2} ; \frac{s-t}{s}\right),
$$

where $F$ denotes the Gauss hypergeometric function. Denote $v=1-\frac{s}{t}$ and let $t$ be fixed. Then

$$
k_{H}(t, s)=k_{H}(t, t(1-v))=t^{H-\frac{1}{2}} v^{H-\frac{1}{2}} F\left(\frac{1}{2}-H, H-\frac{1}{2}, H+\frac{1}{2} ; \frac{v}{v-1}\right),
$$

where $v \in[0,1]$. By $[2$, p. 269, eq. (8.2.9)] and the symmetry of Gauss hypergeometric function with respect to first two parameters, we have

$$
F\left(\frac{1}{2}-H, H-\frac{1}{2}, H+\frac{1}{2} ; \frac{v}{v-1}\right)=(1-v)^{\frac{1}{2}-H} F\left(1, \frac{1}{2}-H, H+\frac{1}{2} ; v\right),
$$

and hence it suffices to show that

$$
\left(\frac{v}{1-v}\right)^{H-\frac{1}{2}} F\left(1, \frac{1}{2}-H, H+\frac{1}{2} ; v\right)
$$


is increasing as a function of $v$. To show this, we use the Euler integral formula [2, p. 271, Proposition 8.3.1.]

$$
F(a, b, c ; v)=\frac{1}{\mathrm{~B}(b, c-b)} \int_{0}^{1} x^{b-1}(1-x)^{c-b-1}(1-v x)^{-a} \mathrm{~d} x
$$

provided that $|v|<1$ and $c>b>0$, where $\mathrm{B}(a, b)$ denotes the Beta function. Hence we have

$$
\begin{aligned}
& \left(\frac{v}{1-v}\right)^{H-\frac{1}{2}} F\left(1, \frac{1}{2}-H, H+\frac{1}{2} ; v\right) \\
& =\frac{1}{\mathrm{~B}\left(1, H-\frac{1}{2}\right)}\left(\frac{v}{1-v}\right)^{H-\frac{1}{2}} \int_{0}^{1}(1-x)^{H-\frac{3}{2}}(1-v x)^{H-\frac{1}{2}} \mathrm{~d} x \\
& =\frac{1}{\mathrm{~B}\left(1, H-\frac{1}{2}\right)} \int_{0}^{1}(1-x)^{H-\frac{3}{2}}\left(\frac{v}{1-v}(1-v x)\right)^{H-\frac{1}{2}} \mathrm{~d} x .
\end{aligned}
$$

Now it is straightforward to see that, for any $x \in(0,1)$,

$$
\frac{v}{1-v}-\frac{v^{2}}{1-v} x
$$

is an increasing function in $v$. Consequently, $k_{H}(t, s)=k_{H}(t, t(1-v))$ is also increasing as a function of $v$, and thus $-k_{H}(t, u) k_{H}(s, u)$ is increasing in $u$, which shows that, for fixed $t$ and $s, \hat{r}_{H}(t, s \mid u)$ is a convex function.

Proof of Proposition 3.2. Denote

$$
\beta_{H}(\tau)=\int_{1}^{\tau} w^{H-\frac{3}{2}}(w-1)^{H-\frac{1}{2}} \mathrm{~d} w
$$

Then, by using the change of variable $w=\frac{z}{v}$ in (2.1) we can write

$$
k_{H}(t, v)=d_{H}\left[\left(\frac{t}{v}\right)^{H-\frac{1}{2}}(t-v)^{H-\frac{1}{2}}-\left(H-\frac{1}{2}\right) v^{H-\frac{1}{2}} \beta_{H}\left(\frac{t}{v}\right)\right] .
$$

Then, from (3.2) it follows that

$$
\begin{aligned}
& \hat{r}_{H}(t, s \mid u)-r_{H}(t, s) \\
& \quad=-d_{H}^{2} \int_{0}^{u}\left[I_{1}^{H}(t, s, v)+I_{2}^{H}(t, s, v)+I_{3}^{H}(t, s, v)+I_{4}^{H}(t, s, v)\right] \mathrm{d} v,
\end{aligned}
$$

where

$$
\begin{aligned}
I_{1}^{H}(t, s, v) & =\left(\frac{t}{v}\right)^{H-\frac{1}{2}}\left(\frac{s}{v}\right)^{H-\frac{1}{2}}(t-v)^{H-\frac{1}{2}}(s-v)^{H-\frac{1}{2}}, \\
I_{2}^{H}(t, s, v) & =\left(\frac{1}{2}-H\right) t^{H-\frac{1}{2}}(t-v)^{H-\frac{1}{2}} \beta_{H}\left(\frac{s}{v}\right), \\
I_{3}^{H}(t, s, v) & =\left(\frac{1}{2}-H\right) s^{H-\frac{1}{2}}(s-v)^{H-\frac{1}{2}} \beta_{H}\left(\frac{t}{v}\right), \\
I_{4}^{H}(t, s, v) & =\left(H-\frac{1}{2}\right)^{2} v^{2 H-1} \beta_{H}\left(\frac{s}{v}\right) \beta_{H}\left(\frac{t}{v}\right) .
\end{aligned}
$$

Consider first the term $I_{1}^{H}(t, s, v)$. Recall that $\left|a^{\gamma}-b^{\gamma}\right| \leq|a-b|^{\gamma}$ for any $\gamma \in(0,1)$. Consequently, for $H>\frac{1}{2}$,

$$
\left|(t-v)^{H-\frac{1}{2}}-t^{H-\frac{1}{2}}\right| \leq v^{H-\frac{1}{2}},
$$


which implies that

$$
(t-v)^{H-\frac{1}{2}}=t^{H-\frac{1}{2}}+O\left(v^{H-\frac{1}{2}}\right) .
$$

Similarly, for $H<\frac{1}{2}$,

$$
\begin{aligned}
\left|(t-v)^{H-\frac{1}{2}}-t^{H-\frac{1}{2}}\right| & =\left|\frac{1}{(t-v)^{\frac{1}{2}-H}}-\frac{1}{t^{\frac{1}{2}-H}}\right| \\
& \leq\left|\frac{1}{t-v}-\frac{1}{t}\right|^{\frac{1}{2}-H} \\
& =\left(\frac{v}{(t-v) t}\right)^{\frac{1}{2}-H} .
\end{aligned}
$$

Consequently, as $v \rightarrow 0$, we have

$$
(t-v)^{H-\frac{1}{2}}=t^{H-\frac{1}{2}}+O\left(v^{\frac{1}{2}-H}\right) .
$$

Hence, as $v \rightarrow 0$,

$$
I_{1}^{H}(t, s, v)=(t s)^{2 H-1} v^{1-2 H}+o\left(v^{1-2 H}\right)
$$

and, consequently,

$$
\int_{0}^{u} I_{1}^{H}(t, s, v) \mathrm{d} v=\frac{(t s)^{2 H-1}}{2-2 H} u^{2-2 H}+o\left(u^{2-2 H}\right) .
$$

Consider then the next three remaining terms $I_{2}^{H}(t, s, v), I_{3}^{H}(t, s, v)$ and $I_{4}^{H}(t, s, v)$. We begin with the case $H<\frac{1}{2}$. Now $\beta_{H}(\infty)<\infty$ and

$$
\begin{aligned}
\left|\beta_{H}\left(\frac{t}{v}\right)-\beta_{H}(\infty)\right| & =\int_{\frac{t}{v}}^{\infty} w^{H-\frac{3}{2}}(w-1)^{H-\frac{1}{2}} \mathrm{~d} w \\
& \leq \int_{\frac{t}{v}}^{\infty}(w-1)^{2 H-2} \mathrm{~d} w \\
& =C_{H}\left(\frac{t}{v}-1\right)^{2 H-1} \\
& \leq C_{H, t} v^{1-2 H}
\end{aligned}
$$

for small enough $v$. Consequently,

$$
\beta_{H}\left(\frac{t}{v}\right)=\beta_{H}(\infty)+O\left(v^{1-2 H}\right) .
$$

By using this together with (4.3) we get

$$
I_{2}^{H}(t, s, v)=\left(\frac{1}{2}-H\right) t^{H-\frac{1}{2}} \beta_{H}(\infty)+O\left(v^{\frac{1}{2}-H}\right)
$$

from which it follows that

$$
\int_{0}^{u} I_{2}^{H}(t, s, v) \mathrm{d} v=O(u)=o\left(u^{2 H}\right) .
$$

Moreover, with the same arguments we observe

$$
\int_{0}^{u} I_{3}^{H}(t, s, v) \mathrm{d} v=o\left(u^{2 H}\right)
$$


and by (4.4) we also have

$$
\int_{0}^{u} I_{1}^{H}(t, s, v) \mathrm{d} v=O\left(u^{2-2 H}\right)=o\left(u^{2 H}\right) .
$$

Finally, for $I_{4}^{H}$ we have, again thanks to (4.5),

$$
I_{4}^{H}(t, s, v)=\left(H-\frac{1}{2}\right)^{2}\left(\int_{1}^{\infty} w^{H-\frac{3}{2}}(w-1)^{H-\frac{1}{2}} \mathrm{~d} w\right)^{2} v^{2 H-1}+O(1)
$$

from which the claim follows by integrating with respect to $v$ over the interval $[0, u]$ for $H<\frac{1}{2}$. Let then $H>\frac{1}{2}$. We have

$$
\begin{aligned}
\beta_{H}\left(\frac{t}{v}\right) & =\int_{1}^{\frac{t}{v}} w^{2 H-2} \mathrm{~d} w+\int_{1}^{\frac{t}{v}} w^{H-\frac{3}{2}}\left[(w-1)^{H-\frac{1}{2}}-w^{H-\frac{1}{2}}\right] \mathrm{d} w \\
& =\frac{t^{2 H-1}}{2 H-1} v^{1-2 H}+O\left(v^{\frac{1}{2}-H}\right),
\end{aligned}
$$

where the last equality follows from (4.1). By using (4.2) again we hence observe that

$$
I_{2}^{H}(t, s, v)=\left(\frac{1}{2}-H\right) \frac{(t s)^{2 H-1}}{2 H-1} v^{1-2 H}+O\left(v^{\frac{1}{2}-H}\right) .
$$

Since $O\left(u^{\frac{3}{2}-H}\right)=o\left(u^{2-2 H}\right)$ for $H>\frac{1}{2}$, we have

$$
\int_{0}^{u} I_{2}^{H}(t, s, v) \mathrm{d} v=\left(\frac{1}{2}-H\right) \frac{(t s)^{2 H-1}}{(2 H-1)(2-2 H)} u^{2-2 H}+o\left(u^{2-2 H}\right) .
$$

Similarly, we observe

$$
\int_{0}^{u} I_{3}^{H}(t, s, v) \mathrm{d} v=\left(\frac{1}{2}-H\right) \frac{(t s)^{2 H-1}}{(2 H-1)(2-2 H)} u^{2-2 H}+o\left(u^{2-2 H}\right) .
$$

For $I_{4}^{H}(t, s, v)$, we obtain by (4.6) that

$$
I_{4}^{H}(t, s, v)=\left(H-\frac{1}{2}\right)^{2} \frac{(t s)^{2 H-1}}{(2 H-1)^{2}} v^{1-2 H}+O\left(v^{\frac{1}{2}-H}\right)
$$

and hence

$$
\int_{0}^{u} I_{4}^{H}(t, s, v) \mathrm{d} v=\left(H-\frac{1}{2}\right)^{2} \frac{(t s)^{2 H-1}}{(2-2 H)(2 H-1)^{2}} u^{2-2 H}+o\left(u^{2-2 H}\right) .
$$

Now the result follows by combining equations (4.7)-(4.9) with (4.4) together with some simplifications.

Proof of Proposition 3.3. Let $\beta_{H}$ and $I_{i}^{H}(t, s, v), i=1,2,3,4$, be like in the proof of Proposition 3.2.

We begin by showing that the terms $I_{2}^{H}(t, s, v)$ and $I_{4}^{H}(t, s, v)$ are negligible. For this note that

$$
\beta_{H}\left(\frac{s}{v}\right) \leq C_{H}\left(\frac{s}{v}-1\right)^{H+\frac{1}{2}} \leq C_{H}(s-v)^{H+\frac{1}{2}}
$$

for $v$ close enough to $s$. Consequently, for $t>s$ we have

$$
I_{2}^{H}(t, s, v)=O\left((s-v)^{H+\frac{1}{2}}\right)
$$

from which it follows that

$$
\int_{u}^{s} I_{2}^{H}(t, s, v) \mathrm{d} v=o\left((s-v)^{H+\frac{1}{2}}\right) .
$$


Similarly, for $t=s$ we have

$$
I_{2}^{H}(s, s, v)=O\left((s-v)^{2 H}\right)
$$

and thus

$$
\int_{u}^{s} I_{2}^{H}(s, s, v) \mathrm{d} v=o\left((s-v)^{2 H}\right) .
$$

This implies that the term $I_{2}^{H}(t, s, v)$ is negligible. For terms $I_{3}^{H}(t, s, v)$ and $I_{4}^{H}(t, s, v)$, we first observe that

$$
\beta_{H}\left(\frac{t}{v}\right)=\beta_{H}\left(\frac{t}{s}\right)+\int_{\frac{t}{s}}^{\frac{t}{v}} w^{H-\frac{3}{2}}(w-1)^{H-\frac{1}{2}} \mathrm{~d} w .
$$

Here the first term, denoted by $\beta_{H}\left(\frac{t}{s}\right)$, is just a constant independent of $v$ and $u$. For the second term we have

$$
\int_{\frac{t}{s}}^{\frac{t}{v}} w^{H-\frac{3}{2}}(w-1)^{H-\frac{1}{2}} \mathrm{~d} w \leq C_{H, t, s}\left(\frac{t}{v}-\frac{t}{s}\right)^{H+\frac{1}{2}} \leq C_{H, t, s}(s-v)^{H+\frac{1}{2}}
$$

for $v$ close to $s$. Hence the term $I_{4}^{H}(t, s, v)$ is also negligible. Indeed, combining estimates (4.10) and (4.12) we get

$$
\int_{u}^{s} I_{4}^{H}(t, s, v) \mathrm{d} v \leq C_{H, t, s} \int_{u}^{s}\left[\beta_{H}\left(\frac{t}{s}\right)(s-v)^{H+\frac{1}{2}}+(s-v)^{2 H+1}\right] \mathrm{d} v .
$$

Consequently, for $t>s$ we have

$$
\int_{u}^{s} I_{4}^{H}(t, s, v) \mathrm{d} v=o\left((s-u)^{H+\frac{1}{2}}\right)
$$

and for $t=s$, thanks to the fact $\beta_{H}(1)=0$, we have

$$
\int_{u}^{s} I_{4}^{H}(s, s, v) \mathrm{d} v=o\left((s-u)^{2 H}\right) .
$$

Let us next study the term $I_{3}^{H}(t, s, v)$. By using the decomposition (4.11) and the estimate (4.12) we obtain that

$$
I_{3}^{H}(t, s, v)=\left(\frac{1}{2}-H\right) s^{H-\frac{1}{2}}(s-v)^{H-\frac{1}{2}} \beta_{H}\left(\frac{t}{s}\right)+O\left((s-v)^{2 H}\right) .
$$

Hence for $t>s$ we have

$$
\int_{u}^{s} I_{3}^{H}(t, s, v) \mathrm{d} v=\frac{\frac{1}{2}-H}{\frac{1}{2}+H} \beta_{H}\left(\frac{t}{s}\right) s^{H-\frac{1}{2}}(s-u)^{H+\frac{1}{2}}+o\left((s-u)^{H+\frac{1}{2}}\right)
$$

and for $t=s$ we have

$$
\int_{u}^{s} I_{3}^{H}(s, s, v) \mathrm{d} v=o\left((s-u)^{2 H}\right) .
$$

To conclude the proof, it remains to study the term $I_{1}^{H}(t, s, v)$. We write

$$
I_{1}^{H}(t, s, v)=\left(\frac{t}{s}\right)^{H-\frac{1}{2}}\left(\frac{s}{v}\right)^{2 H-1}(t-v)^{H-\frac{1}{2}}(s-v)^{H-\frac{1}{2}} .
$$

Furthermore, using similar analysis as above we observe that, for $v$ close to $s$, we have

$$
\left(\frac{s}{v}\right)^{2 H-1}-1 \leq C_{H}(s-v)^{2 H-1}
$$


for $H>\frac{1}{2}$ and

$$
\left(\frac{s}{v}\right)^{2 H-1}-1 \leq C_{H}(s-v)^{1-2 H}
$$

for $H<\frac{1}{2}$. Thus instead of $I_{1}^{H}(t, s, v)$ it suffices to consider

$$
\left(\frac{t}{s}\right)^{H-\frac{1}{2}}(t-v)^{H-\frac{1}{2}}(s-v)^{H-\frac{1}{2}}
$$

from which we easily observe that, for $t=s$, we have

$$
\int_{u}^{s} I_{1}^{H}(t, s, v) \mathrm{d} v=\frac{1}{2 H}\left(\frac{t}{s}\right)^{H-\frac{1}{2}}(s-u)^{2 H}+o\left((s-u)^{2 H}\right) .
$$

For $t>s$ we write

$$
\begin{aligned}
\int_{u}^{s}(t & -v)^{H-\frac{1}{2}}(s-v)^{H-\frac{1}{2}} \mathrm{~d} v \\
= & \int_{u}^{s}(t-s)^{H-\frac{1}{2}}(s-v)^{H-\frac{1}{2}} \mathrm{~d} v \\
& \quad+\int_{u}^{s}\left[(t-v)^{H-\frac{1}{2}}-(t-s)^{H-\frac{1}{2}}\right](s-v)^{H-\frac{1}{2}} \mathrm{~d} v \\
= & \frac{1}{H+\frac{1}{2}}(t-u)^{H-\frac{1}{2}}(s-u)^{H+\frac{1}{2}} \\
& \quad+\int_{u}^{s}\left[(t-v)^{H-\frac{1}{2}}-(t-s)^{H-\frac{1}{2}}\right](s-v)^{H-\frac{1}{2}} \mathrm{~d} v .
\end{aligned}
$$

For $H>\frac{1}{2}$ we have

$$
\left|(t-v)^{H-\frac{1}{2}}-(t-s)^{H-\frac{1}{2}}\right| \leq(s-v)^{H-\frac{1}{2}},
$$

from which it follows that

$$
\int_{u}^{s}\left[(t-v)^{H-\frac{1}{2}}-(t-s)^{H-\frac{1}{2}}\right](s-v)^{H-\frac{1}{2}} \mathrm{~d} v=O\left((s-u)^{2 H}\right)=o\left((s-u)^{H+\frac{1}{2}}\right) .
$$

Similarly, for $H<\frac{1}{2}$ we have

$$
\left|(t-v)^{H-\frac{1}{2}}-(t-s)^{H-\frac{1}{2}}\right| \leq\left(\frac{v-s}{(t-v)(t-s)}\right)^{H-\frac{1}{2}} \leq(t-s)^{2 H-1}(s-v)^{\frac{1}{2}-H} .
$$

Hence

$$
\int_{u}^{s}\left[(t-v)^{H-\frac{1}{2}}-(t-u)^{H-\frac{1}{2}}\right](s-v)^{H-\frac{1}{2}} \mathrm{~d} v=O(s-u)=o\left((s-u)^{H+\frac{1}{2}}\right) .
$$

Combining the above estimates we thus observed that, in the case $t>s$,

$$
\int_{u}^{s} I_{1}^{H}(t, s, v) \mathrm{d} v=\frac{1}{H+\frac{1}{2}}\left(\frac{t}{s}\right)^{H-\frac{1}{2}}(t-s)^{H-\frac{1}{2}}(s-u)^{H+\frac{1}{2}}+o\left((s-u)^{H+\frac{1}{2}}\right) .
$$




\section{REFERENCES}

[1] E. Azmoodeh, T. Sottinen, L. VittasaAri, And A. Yazigi, Necessary and sufficient conditions for Hölder continuity of Gaussian processes, Statist. Probab. Lett., 94 (2014), pp. 230235.

[2] R. Beals And R. Wong, Special functions, vol. 126 of Cambridge Studies in Advanced Mathematics, Cambridge University Press, Cambridge, 2010. A graduate text.

[3] F. Biagini, Y. Hu, B. Øksendal, and T. Zhang, Stochastic calculus for fractional Brownian motion and applications, Probability and its Applications (New York), Springer-Verlag London, Ltd., London, 2008.

[4] V. I. Bogachev, Gaussian measures, vol. 62 of Mathematical Surveys and Monographs, American Mathematical Society, Providence, RI, 1998.

[5] G. Gripenberg and I. Norros, On the prediction of fractional Brownian motion, J. Appl. Probab., 33 (1996), pp. 400-410.

[6] S. Janson, Gaussian Hilbert spaces, vol. 129 of Cambridge Tracts in Mathematics, Cambridge University Press, Cambridge, 1997.

[7] C. Jost, Transformation formulas for fractional Brownian motion, Stochastic Process. Appl., 116 (2006), pp. 1341-1357.

[8] T. LaGatta, Continuous disintegrations of Gaussian processes, Theory Probab. Appl., 57 (2013), pp. 151-162.

[9] Y. S. MishURA, Stochastic calculus for fractional Brownian motion and related processes, vol. 1929 of Lecture Notes in Mathematics, Springer-Verlag, Berlin, 2008.

[10] I. Norros, E. Valkeila, And J. Virtamo, An elementary approach to a Girsanov formula and other analytical results on fractional Brownian motions, Bernoulli, 5 (1999), pp. 571-587.

[11] V. Pipiras And M. S. TAQQU, Are classes of deterministic integrands for fractional Brownian motion on an interval complete?, Bernoulli, 7 (2001), pp. 873-897.

[12] S. G. SAmko, A. A. Kilbas, And O. I. Marichev, Fractional integrals and derivatives, Gordon and Breach Science Publishers, Yverdon, 1993. Theory and applications, Edited and with a foreword by S. M. Nikol'skil, Translated from the 1987 Russian original, Revised by the authors.

[13] T. Sottinen and A. Yazigi, Generalized Gaussian bridges, Stochastic Process. Appl., 124 (2014), pp. 3084-3105. 